\title{
INCIDÊNCIA DE FUNGOS EM SEMENTES DE PINHÃO-MANSO ${ }^{1}$
}

\author{
Leimi Kobayasti ${ }^{2}$, Andressa Iraides Adoriam², \\ Vespasiano Borges de Paiva Neto ${ }^{3}$, Charline Zaratin Alves ${ }^{3}$, Mônica Cristina Rezende Zuffo ${ }^{3}$
}

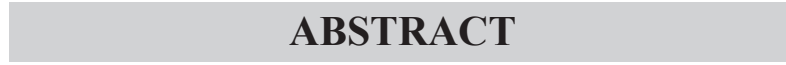

FUNGI INCIDENCE ON Jatropha curcas L. SEEDS

The knowledge on seed pathogens is essential for setting management recommendations. Thus, aiming to determine the pathogens associated with Jatropha curcas L., in order to develop strategies to control them, the blotter test, modified with hydric restrictor $\mathrm{NaCl}-1.0 \mathrm{MPa}$, and evaluation after seven days of incubation, at $25^{\circ} \mathrm{C}$, with a 12 -hours photoperiod, was used. The Jatropha seeds evaluated presented a high level of fungal species, regardless of their surface disinfection. Nineteen different fungi genera were identified, especially Alternaria alternata, Colletotrichum sp., Fusarium spp., and Rhizoctonia solani, which are commonly pathogenic and whose relevance must be taken into account.

KEY-WORDS: Jatropha curcas L.; blotter test; pathogenic fungi.

\section{INTRODUÇÃO}

O pinhão-manso (Jatropha curcas L.) é uma planta oleaginosa tropical e bem adaptada a diversas regiões do Brasil, sendo seu plantio incentivado, nos últimos anos, como alternativa ao fornecimento de matéria-prima para a fabricação de biodiesel. Adicionalmente à capacidade de produzir óleo vegetal, a planta é tolerante ao déficit hídrico, menos exigente em nutrientes e apresenta capacidade de recuperação de áreas degradadas, por possuir raízes profundas e que crescem em solos de baixa fertilidade (Teixeira 2005).

Devido às suas vantagens, verifica-se crescente demanda por sementes de boa qualidade fisiológica e sanitária, e, para isto, deve ser avaliada a ocorrência de patógenos nas mesmas. A semente de pinhão-manso é constituída por tegumento (25\%) denso e escuro, envolvendo o embrião $(75 \%)$, rico

\section{RESUMO}

O conhecimento de patógenos associados a sementes é essencial para a elaboração de recomendações de manejo de uma cultura. Dessa forma, objetivou-se determinar os patógenos associados a sementes de pinhão-manso, para possibilitar o desenvolvimento de estratégias de controle dos mesmos. Para o teste de sanidade, utilizou-se o método do papel de filtro (blotter test), modificado com restritor hídrico $\mathrm{NaCl}-1,0 \mathrm{MPa}$, e avaliação após sete dias de incubação, a $25^{\circ} \mathrm{C}$, com fotoperíodo de 12 horas. As sementes de pinhão-manso analisadas apresentaram alta frequência de espécies fúngicas, independentemente da desinfestação superficial das mesmas. Foram observados 19 gêneros de fungos, destacando-se Alternaria alternata, Colletotrichum sp., Fusarium spp. e Rhizoctonia solani, os quais são comumente patogênicos e devem ter sua relevância considerada.

PALAVRAS-CHAVE: Jatropha curcas L.; teste de sanidade; fungos fitopatogênicos.

em óleo. Apresenta, ainda, pequena variação de tamanho e densidade, sem problemas de dormência (Shepetina \& Sevast'yanova 1986).

Inúmeros danos podem ser provocados por patógenos associados a sementes, dentre eles, a morte em pré-emergência, podridão radicular, tombamento de mudas, manchas necróticas em folhas, caules e frutos, deformações, como hipertrofias e subdesenvolvimento, descoloração de tecidos, infecções latentes e outros (Neergaard 1977). Outros danos podem ser provocados na própria semente, como podridão e perdas do poder germinativo. Para culturas agronômicas, estes danos resultam em redução do stand e da produtividade (Machado 1988). Assim, a semente contaminada ou infectada é um dos meios mais eficientes de introdução, disseminação e acúmulo de inóculo de patógenos, em áreas de cultivo (Neergaard 1977), além de ser eficiente meio de

1. Trabalho recebido em ago./2010 e aceito para publicação em set./2011 (n registro: PAT 10948/ DOI: 10.5216/pat.v41i3.10948).

2. Universidade Federal do Mato Grosso, Faculdade de Agronomia e Medicina Veterinária, Cuiabá, MT, Brasil.

E-mails: leimikobayasti@yahoo.com.br, andressa_adoriam@hotmail.com.

3. Universidade Federal do Mato Grosso do Sul, Chapadão do Sul, MS̄, Brasil.E-mails: vespasiano@nin.ufms.br, charline@ufms.br, mczuffo@nin.ufms.br. 
sobrevivência de patógenos na natureza (Massola Júnior \& Bedendo 2005).

Segundo Aguiar et al. (2001), sementes são eficientes meios de disseminação e de introdução de patógenos em áreas isentas. $\mathrm{O}$ inóculo inicial de epidemias pode depender da transmissão do patógeno pela semente, assim como reduzir a qualidade fisiológica da mesma. Danos decorrentes da associação de micro-organismos patogênicos com sementes não se limitam a perdas diretas da população em campo, mas envolvem outras implicações, que podem provocar sérios danos, em todo o sistema de produção (Machado 1994, Marcos Filho 2001).

Os resultados de pesquisas com a cultura do pinhão-manso são ainda incipientes e preliminares, principalmente com relação a doenças. Devido à atual expansão da área cultivada com esta espécie, há necessidade de maiores informações sobre os patógenos e as doenças potenciais que os mesmos causam à cultura. Desta forma, este trabalho teve como objetivo determinar os patógenos associados a sementes de pinhão-manso, com a finalidade de se obter informações para o estabelecimento de futuras estratégias de controle de doenças, em áreas de produção.

\section{MATERIAL E MÉTODOS}

O experimento foi conduzido por meio de parceria entre a Universidade Federal do Mato Grosso do Sul (UFMS), Campus de Chapadão do Sul, e a Universidade Federal do Mato Grosso (UFMT), Campus de Cuiabá. Os frutos de pinhão-manso foram coletados em julho de 2009, em plantas do Banco de Germoplasma ex situ estabelecido em 2006, em área cedida pela Fundação de Apoio à Pesquisa Agropecuária de Chapadão, localizada no município de Chapadão do Sul (MS).

Após a coleta, as sementes foram removidas dos frutos e o lote maduro foi colocado para secar à temperatura ambiente $\left(27^{\circ} \mathrm{C} \pm 5^{\circ} \mathrm{C}\right)$ e à sombra, e, em seguida, enviadas para o Laboratório de Fitopatologia da Faculdade de Agronomia e Medicina Veterinária da UFMT, onde as análises de sanidade das sementes foram realizadas, em agosto de 2009.

As amostras de sementes de pinhão-manso foram divididas em quatro lotes (tratamentos): fruto maduro (pericarpo amarelo) com desinfestação; fruto maduro sem desinfestação; fruto seco (pericarpo escuro e desidratado) com desinfestação; e fruto seco sem desinfestação.
Os tratamentos com desinfestação foram realizados com imersão das sementes em hipoclorito de sódio ( $1,0 \%$ de cloro ativo), por 20 minutos, seguido de etanol $70 \%(\mathrm{v} / \mathrm{v})$, por 2 minutos. O teste de sanidade das sementes foi realizado com o método do papel de filtro (blotter test), modificado com restrição hídrica, utilizando-se, como soluto, $\mathrm{NaCl}-1,0 \mathrm{MPa}$.

As sementes foram distribuídas uniformemente em placas de Petri de $15 \mathrm{~cm}$ de diâmetro, tendo como substrato três folhas de papel de filtro esterilizadas e umedecidas com solução de $\mathrm{NaCl}$. Em seguida, foram incubadas em $\mathrm{BOD}$, à temperatura de $25^{\circ} \mathrm{C} \pm 2^{\circ} \mathrm{C}$, e mantidas, por sete dias, sob regime alternado de 12 horas de luz. Após este período, foi feita a identificação dos fungos, com base em suas características morfológicas (Barnett \& Hunter 1987, Alexopoulos et al. 1996), e quantificada a incidência.

Adotou-se o delineamento inteiramente casualizado, com diferentes números de repetições. Para sementes oriundas de frutos maduros, foram realizadas cinco repetições, com 20 sementes/placa. Para as sementes oriundas de frutos secos, foram efetuadas dez repetições, com 20 sementes/placa.

Os dados foram submetidos a análise de variância e as médias comparadas pelo teste Tukey $(p<0,05)$, utilizando-se o programa estatístico Sisvar (Ferreira 2002).

\section{RESULTADOS E DISCUSSÃO}

Nas análises de sementes de pinhão-manso, foram detectados 19 gêneros de fungos (Tabela 1), com destaque para Alternaria alternata, Colletotrichum gloeosporioides, Penicillium sp. e Rhizoctonia solani, além das espécies de Fusarium (Tabela 1).

Sementes sem desinfestação apresentaram maior percentagem de infestação e maior diversidade de fungos, provavelmente localizados nas camadas mais externas da semente (Figuras 1A e 1B). Sá et al. (2009) detectaram a presença de Colletotrichum, Curvularia, Verticillium, Fusarium, Penicillium, Aspergillus e Alternaria, restrita ao tegumento das sementes de pinhão-manso de diferentes procedências. Mira \& Sierra (2008) relataram a presença de Fusarium sp. e Rhizoctonia sp. em frutos, ramos e folhas de pinhão-manso cultivado na Colômbia. Melo et al. (2007) observaram diversificada incidência de fungos em sementes desinfestadas de pinhão-manso, como Monilia sp., Fusarium sp., Rhizopus sp., Trichoderma sp., Aspergillus spp, Curvularia sp., 
Tabela 1. Incidência de fungos (\%) em sementes desinfestadas (SD) e sem desinfestação (SSD), em frutos secos e maduros de pinhão-manso, provenientes de Chapadão do Sul, MS (Cuiabá, MT, 2009).

\begin{tabular}{|c|c|c|c|c|}
\hline \multirow[t]{2}{*}{ Fungos } & \multicolumn{2}{|c|}{$\begin{array}{c}\text { Frutos } \\
\text { secos }\end{array}$} & \multicolumn{2}{|c|}{$\begin{array}{c}\text { Frutos } \\
\text { maduros }\end{array}$} \\
\hline & SD & SSD & SD & SSD \\
\hline Alternaria alternata & $2,5 \mathrm{~b}$ & $9,5 \mathrm{a}$ & - & $8,0 \mathrm{a}$ \\
\hline Absidia sp. & $1,0 \mathrm{~b}$ & $12,0 \mathrm{a}$ & - & - \\
\hline Acremonium sp. & $1,0 \mathrm{a}$ & $0,5 \mathrm{a}$ & - & - \\
\hline Ascochyta sp. & - & 22,0 & - & - \\
\hline Aspergillus ochraceus & - & - & 7,0 & - \\
\hline Aspergillus sp. & - & - & 12,0 & - \\
\hline Bipolaris sp. & $1,0 \mathrm{a}$ & - & - & $3,0 \mathrm{a}$ \\
\hline Chaetomium sp. & $1,0 \mathrm{~b}$ & $29,0 \mathrm{a}$ & - & - \\
\hline Cladosporium cladosporioides & $4,5 \mathrm{~d}$ & $43,5 \mathrm{~b}$ & $12,0 \mathrm{c}$ & $85,0 \mathrm{a}$ \\
\hline Colletotrichum gloeosporioides & - & 18 & - & - \\
\hline Curvularia sp. & - & - & - & 1,0 \\
\hline Epicoccum sp. & - & - & - & 13,0 \\
\hline Fusarium graminearum & - & $31,5 \mathrm{a}$ & - & $1,0 \mathrm{~b}$ \\
\hline Fusarium pallidoroseum & $1,5 \mathrm{~b}$ & $1,0 \mathrm{~b}$ & - & $26,0 \mathrm{a}$ \\
\hline Fusarium sp. & $2,5 \mathrm{~b}$ & - & - & $11,0 \mathrm{a}$ \\
\hline Fusarium verticillioides & - & $2,5 \mathrm{c}$ & $27,0 \mathrm{a}$ & $11,0 \mathrm{~b}$ \\
\hline Nigrospora sp. & - & - & - & 3,0 \\
\hline Penicillium sp. & $2,0 \mathrm{~b}$ & - & $26,0 \mathrm{a}$ & $6,0 \mathrm{~b}$ \\
\hline Periconia sp. & - & $1,5 \mathrm{~b}$ & - & $17,0 \mathrm{a}$ \\
\hline Pestalotia sp. & - & - & - & 1,0 \\
\hline Phomopsis sp. & - & - & - & 2,0 \\
\hline Rhizoctonia solani & - & 2,5 & - & - \\
\hline Trichothecium sp. & $1,0 \mathrm{a}$ & - & $1,0 \mathrm{a}$ & $2,0 \mathrm{a}$ \\
\hline
\end{tabular}

Médias seguidas de mesma letra, na linha, não diferem significativamente, pelo teste Tukey $(\mathrm{p}<0,05)$.

Alternaria spp., Chaetomium sp e Penicillium sp., em ordem decrescente de incidência. Já em sementes não desinfestadas, os fungos detectados, pelos mesmos autores, foram Aspergillus sp. (57\%), Fusarium sp. (39\%), Colletotrichum sp. (31\%), Rhizopus sp. (27\%), Monilia sp. (24\%), Alternaria sp. (16\%) e Penicillium sp. (6\%).
Popinigis (1977) ressaltou que as espécies dos gêneros Aspergillus sp. e Penicillium sp. se encontram entre os principais agentes deterioradores de sementes. Neves et al. (2009), trabalhando com sementes de pinhão-manso, observaram que sementes com alta incidência conjunta de Fusarium, Aspergillus e Penicillium apresentaram baixo poder de germinação.

Os fungos dos gêneros Aspergillus e Penicillium geralmente estão presentes em sementes recém-colhidas, em percentagens muito baixas, e são capazes de sobreviver em ambientes com baixa umidade, proliferando-se em sucessão aos fungos de campo e causando a deterioração das sementes, culminando com a perda da viabilidade e do valor comercial das mesmas (Berjak 1987, Carvalho \& Nakagawa 1988).

Goldfarb et al. (2010) afirmaram que a crioconservação não reduziu a incidência de patógenos presentes em sementes de pinhão-manso armazenadas. Nas temperaturas criogênicas, ocorreu a preservação da integridade do material biológico, devido à redução das taxas metabólicas, sendo a micoflora presente também preservada. Os fungos de armazenamento observados com maior frequência, após a técnica de crioconservação, foram os pertencentes aos gêneros Aspergillus sp., seguidos de Cladosporium sp. e Fusarium sp.

A presença de Alternaria alternata (Figuras 1B e 1C), que causa a alternariose ou mancha de alternaria, encontrada em frutos secos e maduros, foi de $2,5 \%$ e $9,0 \%$, respectivamente. A ocorrência da associação de sementes com $A$. alternata pode produzir grandes prejuízos, pelo fato de causar infecções em
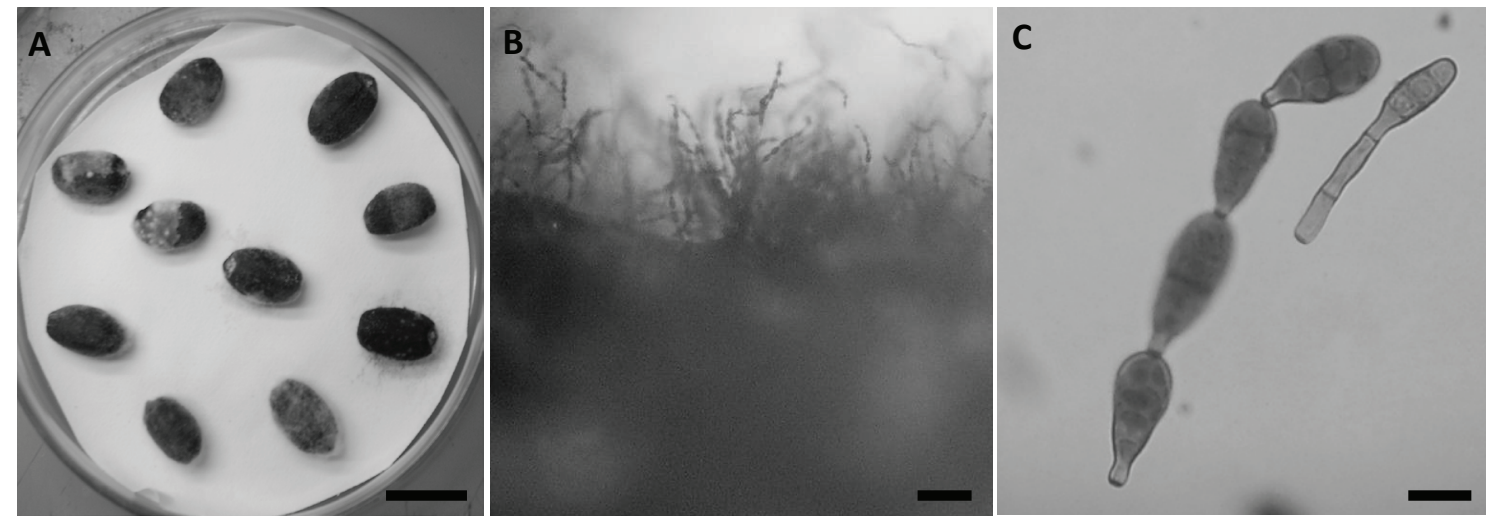

Figura 1. Imagens ilustrativas do experimento: A - presença de fungos na superfície das sementes (Barra = $2 \mathrm{~cm})$; B - Alternaria alternata presente na superfície do tegumento e observado em estereomicroscópio (Barra $=200 \mu \mathrm{m}$ ); $\mathrm{C}-$ Alternaria alternata observado em microscópio óptico $($ Barra $=10 \mu \mathrm{m})$ (Cuiabá, MT, 2009). 
sementes e ser por elas transmitido (Neergaard 1977). Além disto, este fungo causa redução na germinação e tombamento de plântulas (Rotem 1995), manchas foliares e queda prematura de folhas, na cultura do pinhão-manso (Franco \& Gabriel 2008).

O fungo Colletotrichum gloeosporioides foi observado apenas em frutos secos e sem desinfestação, com incidência de $18 \%$. O gênero Colletotrichum é frequentemente relatado em diferentes espécies de plantas cultivadas em todo o mundo, causando doenças e/ou de forma saprófita (Lopez 2001). Em pinhão-manso, a doença conhecida como antracnose é caracterizada por manchas foliares, que podem evoluir para a queima completa das folhas. Os frutos também podem ser infectados com lesões de coloração marrom escura (Franco \& Gabriel 2008). No Brasil, a doença já foi relatada nos Estados do Ceará e Mato Grosso do Sul (Roese \& Freire 2009).

Os fungos Alternaria alternata, Colletotrichum gloeosporioides e Rhizoctonia solani são reconhecidamente patogênicos, possuindo, portanto, potencial para provocar processos de interferência fisiológica em pinhão-manso. A doença conhecida como "tombamento" pode ter, como agente causal, diversos patógenos, dentre eles Rhizoctonia solani, Colletotrichum gloeosporioides e Fusarium spp., todos observados nos lotes analisados. Rhizoctonia solani foi encontrado em apenas $2,5 \%$ das sementes secas não desinfestadas, considerada uma percentagem baixa. De acordo com Lima et al. (2001), fungos do gênero Rhizoctonia sp. podem causar tombamento de plântulas de mamoneira. Vale ressaltar que, embora as sementes tenham sido obtidas de frutos colhidos diretamente nas plantas matrizes, portanto sem contato direto com o solo, a presença de Rhizoctonia solani pode ser atribuída à presença de ventos intensos na região de cultivo, resultando em constantes suspensões de solo, ou ainda durante o processo de secagem, no qual as sementes ficaram expostas em bancadas.

Nas sementes não desinfestadas, foi observada a presença de Fusarium pallidoroseum (26\%) e Fusarium graminearum (31,5\%), provenientes de frutos maduros e frutos secos, respectivamente. Nas sementes desinfestadas e provenientes de frutos maduros, a presença de Fusarium verticillioides (27\%) pode ser explicada por sua localização interna, na semente. As espécies de Fusarium são comumente associadas a sementes de diversas culturas, ocasionando perda de germinação e vigor, e auxiliando, também, no complexo de patógenos que causam tombamento em plantas. Segundo Sharma et al. (2001) e Gour (2006), F. verticillioides já foi encontrado associado à podridão de raízes de pinhão-manso, na Índia. Também já foi relatada a presença de Fusarium sp., associada à podridão seca dos ramos: pequenos ramos com folhas jovens nas pontas secam e quebram facilmente (Padilha \& Monterroso 1999).

Outros fungos observados, como Penicillium sp. e Aspergillus spp., são considerados típicos de armazenamento e podem causar depreciação de grãos ou sementes, principalmente quando armazenadas sob condições inadequadas. Além destes fungos, Chaetomium sp. e Absidia sp. estão, também, associados à deterioração de sementes e sua ação é dependente das condições físicas e fisiológicas das mesmas, por ocasião do início do armazenamento e dos fatores ambientais predominantes no decorrer deste período (Ruiz Filho et al. 2004). Santos et al. (2009), ao avaliarem a qualidade sanitária de sementes de pinhão-manso produzidas no norte de Minas Gerais, por meio do método do papel de filtro (blotter test) e meio de cultura BDA, detectaram os gêneros Fusarium, Aspergillus, Penicillium e Colletotrichum, corroborando os dados apresentados na Tabela 1.

Cladosporium cladosporioides foi observado com percentagens variando de $4,5 \%$ a $85 \%$, com maiores valores nos tratamentos sem desinfestação. As espécies de Cladosporium ocorrem em inúmeras espécies vegetais, especialmente como componente da micoflora de sementes. Além da perda de vigor, certas espécies de fungos, como Cladosporium cladosporioides, são passíveis de causar manchas no tegumento, resultando em aspecto indesejável, e provocam a depreciação dos lotes (Neergaard 1980). Este fungo tem sido relatado como agente patogênico, em diversas culturas, como maracujá (Fischer et al. 2005), tomate (Kurozawa \& Pavan 2005) e mamão (Silva \& Soares 2001).

Bipolaris sp., Phomopsis sp. e Pestalotia sp. foram observados em baixa percentagem (1-3\%). Além destes, Ascochyta sp. (22\%) é considerado patogênico a diversas espécies hospedeiras, causando prejuízos consideráveis. Em sementes, apresenta importância variável, dependendo do hospedeiro e da espécie encontrada, como, por exemplo, em ervilha (Ascochyta sp.), gramíneas (Bipolaris sp.), soja (Phomopsis sp.) e frutíferas (Pestalotia sp.) (Kimati et al. 2005). 
Fungos como Acremonium sp., Chaetomium sp., Curvularia sp., Periconia sp., Epicoccum sp., Nigrospora sp., Trichothecium sp. e Absidia sp. são considerados saprófitas e sempre estão presentes nas sementes, podendo afetar o vigor destas. Machado (1988) relatou que fungos transmissíveis ou não por sementes podem afetar o vigor destas no campo. Assim, o uso de sementes com baixo vigor pode ter reflexos negativos dos mais variáveis, considerando-se não somente o baixo desempenho das plantas, em termos de stand, como, também, a maior vulnerabilidade destas ao ataque de patógenos, conforme relatado por Neves et al. (2009).

Observou-se que, entre os tratamentos com e sem desinfestação, as sementes desinfestadas apresentaram menor percentagem de fungos e menor número de gêneros presentes. Este resultado concorda com Melo et al. (2007), que observaram, em sementes desinfestadas de pinhão-manso, menor incidência de fungos pertencentes aos gêneros encontrados, e discorda do estudo de Vanzolini et al. (2010), no qual não foi constatada diferença acentuada na incidência de fungos entre as sementes desinfestadas ou não com hipoclorito de sódio.

Os fungos Alternaria alternata, Fusarium pallidoroseum, Acremonium sp., Chaetomium sp. e Cladosporium cladosporioides foram observados nos dois tratamentos aplicados em frutos secos, sendo em menor percentagem nos frutos desinfestados, enquanto, em frutos maduros, Fusarium verticillioides, Penicillium sp., Aspergillus sp. e Cladosporium cladosporioides foram os mais representativos, com taxas variando de $12 \%$ a $27 \%$. Estes fungos, provavelmente, estavam alojados internamente à semente, visto que a assepsia tende a eliminar boa parte do inóculo presente externamente. Segundo Machado (1988), um mesmo patógeno pode estar presente em um lote, ou em uma única semente, sob uma ou mais formas de localização. As sementes analisadas apresentam micoflora bastante diversificada, o que pode comprometer a sua qualidade para o plantio. Moreira (2010) observou a associação constante nas sementes de quatro diferentes oleaginosas dos fungos Aspergillus niger, Fusarium sp., Penicillium sp., Rhizoctonia solani, Rhizopus stolonifer e Sclerotium sp., sendo, esta associação mantida em todos os municípios avaliados, independentemente da oleaginosa (mamona, girassol, amendoim e gergelim).

Fungos fitopatogênicos podem associar-se às sementes, em todas as etapas de produção. Esta associação é responsável, muitas vezes, pela redução da qualidade fisiológica das sementes, dispersão de patógenos a longas distâncias e transmissão destes da semente à planta, bem como em meio eficiente de sobrevivência do patógeno, em contato direto com o hospedeiro (Machado 1982).

\section{CONCLUSÕES}

1. Sementes de pinhão-manso apresentaram alta incidência de espécies fúngicas, mesmo após a desinfestação superficial.

2. Os fungos Alternaria alternata, Colletotrichum gloeosporioides, Penicillium sp. e Rhizoctonia solani, além das espécies de Fusarium, detectados em sementes de pinhão-manso, são potencialmente patogênicos e devem ter sua relevância considerada.

\section{REFERÊNCIAS}

AGUIAR, R. H. et al. Qualidade física, fisiológica e sanitária de sementes de girassol de diferentes tamanhos. Revista Brasileira de Sementes, Londrina, v. 23, n. 1, p. 134-139, 2001.

ALEXOPOULOS, C. J.; MIMS, C. W.; BLACKWELL, M. Introductory mycology. 4. ed. New York: John Wiley \& Sons, 1996.

BARNETT, H. L.; HUNTER, B. B. Ilustrated genera of imperfect fungi. 4. ed. New York: Macmillan, 1987.

BERJAK, P. Stored seeds: the problems caused by microorganisms. In: BERJAK, P. (Ed.). Advanced international course on seed phatology. Passo Fundo: Embrapa/Abrates, 1987. p. 93-112.

CARVALHO, N. M.; NAKAGAWA, J. Sementes: ciência, tecnologia e produção. 3. ed. Campinas: Fundação Cargill, 1988.

FERREIRA, D. F. Sisvar: sistema de análise de variância para dados balanceados, versão 4.3. Lavras: UFLa, 2002.

FISCHER, I. H.; KIMATI, H.; REZENDE, J. A. M. Doenças do maracujazeiro (Passiflora spp.). In: KIMATI, H. et al. (Eds.). Manual de fitopatologia: doenças das plantas cultivadas. 4. ed. São Paulo: Agronômica Ceres, 2005. p. 468-474.

FRANCO, D. A. S.; GABRIEL, D. Aspectos fitossanitários na cultura do pinhão-manso (Jatropha curcas L.) para produção de biodiesel. Biológico, São Paulo, v. 70, n. 1, p. 63-64, 2008. 
GOLDFARB, M. et al. Incidência de fungos e qualidade fisiológica de sementes de pinhão manso (Jatropha curcas L.) após o armazenamento criogênico. Biotemas, Florianópolis, v. 23, n. 1, p. 19-26, 2010.

GOUR, V. K. Production practices including post harvest management of Jatropha curcas. In: SINGH, B.; SWAMINATHAN, R.; PONRAJ, V. (Eds.). Biodiesel conference towards energy independence: focus on Jatropha. New Delhi: Rashtrapati Bhawan, 2006. p. 223-251.

KIMATI, H. et al. (Eds.). Manual de fitopatologia: doenças das plantas cultivadas. 4. ed. São Paulo: Agronômica Ceres, 2005.

KUROZAWA, C.; PAVAN, M. A. Doenças do tomateiro (Lycopersicum esculentum). In: KIMATI, H. et al. (Eds.). Manual de fitopatologia: doenças das plantas cultivadas. 4. ed. São Paulo: Agronômica Ceres, 2005. p. 607-626.

LIMA, E. F.; ARAÚJO, A. E.; BATISTA, F. A. S. Doenças e seu controle. In: AZEVEDO, D. M. P.; LIMA. E. F. (Eds.). O agronegócio da mamona no Brasil. Brasília, DF: Embrapa Informação Tecnológica, 2001. p. 190-212.

LOPEZ, A. M. Q. Taxonomia, patogênese e controle de espécies do gênero Colletotrichum. Revisão Anual de Patologia de Plantas, Passo Fundo, v. 9, n. 1, p. 291-338, 2001.

MACHADO, J. C. Controle de fitopatógenos associados a sementes. Informe Agropecuário, Belo Horizonte, v. 8, n. 91 , p. 34-40, 1982.

MACHADO, J. C. Patologia de sementes: fundamentos e aplicações. Brasília, DF: Ministério da Educação, 1988.

MACHADO, J. C. Podridões de tolerância de patógenos associados a sementes. Revisão Anual de Patologia de Plantas, Passo Fundo, v. 2, n. 1, p. 229-263, 1994.

MARCOS FILHO, J. Pesquisa sobre vigor de sementes de hortaliças. Informativo Abrates, Londrina, v. 11, n. 1, p. 63-75, 2001.

MASSOLA JÚNIOR, N. S.; BEDENDO, I. P. Doenças da mamoneira (Ricinus communis L.). In: KIMATI, M. et al. Manual de fitopatologia: doenças das plantas cultivadas. 4. ed. São Paulo: Agronômica Ceres, 2005. p. 445-447.

MELO, M. F. V. et al. Fungos associados a sementes de pinhão manso (Jatropha curcas L.). 2007. Disponível em: <http://www.biodiesel.gov.br/docs/congresso2007/ agricultura/46.pdf $>$. Acesso em: 26 jan. 2011.

MIRA, G. E. M.; SIERRA, J. F. S. Obtención de biodiesel a partir del aceite de la semilla de Jatropha curcas L. (piñón) y evaluación de los parámetros autoecológicos de la planta. 2008. 112 f. Trabalho de Conclusão de Curso (Graduação em Engenharia Ambiental)-Universidad de Medellín, Medelín, 2008.

MOREIRA, F. J. C. Fungos associados às oleaginosas mamona, girassol, amendoim e gergelim na região do Cariri, no Estado do Ceará. In: CONGRESSO BRASILEIRO DE MAMONA, 4.; SIMPÓSIO INTERNACIONAL
DE OLEAGINOSAS ENERGÉTICAS, 1., 2010, João Pessoa. Anais... Campina Grande: Embrapa Algodão, 2010. p. 996-1001.

NEERGAARD, P. A review on quarantine for seed. Copenhagen: CNAS, 1980.

NEERGAARD, P. Seed pathology. London: The MacMillan Press, 1977.

NEVES, W. S. et al. Avaliação fitossanitária de sementes de pinhão-manso provenientes dos Vales do Jequitinhonha e Mucuri. Revista Trópica, Chapadinha, v. 3, n. 2, p. 1723, 2009.

PADILHA, D.; MONTERROSO, D. Diagnostico preliminar de enfermedades del cultivo de tempate (Jatropha curcas) en Nicaragua. Manejo Integrado de Plagas, Turrialba, v. 51, n. 1, p. 66-69, 1999.

POPINIGIS, F. Fisiologia da semente. Brasília, DF: Ministério da Agricultura, 1977.

ROESE, A. D.; FREIRE, F. C. O. Doenças do pinhãomanso (Jatropha curcas L.). In: SIMPÓSIO DE CONTROLE DE DOENÇAS DE PLANTAS, 9., 2009, Lavras. Anais... Lavras: UFLa, 2009. p. 175-183.

ROTEM, J. The genus Alternaria. St. Paul: The American Phytopathological Society, 1995.

RUIZ FILHO, R. R. et al. Fungos associados às sementes de cedro. Summa Phytopathologica, Botucatu, v. 30, n. 4, p. 494-496, 2004.

SÁ, D. A. C. et al. Identificação de fungos externa e internamente a sementes de pinhão-manso. In: CONGRESSO BRASILEIRO DE PESQUISA EM PINHÃO-MANSO, 1., 2009, Brasília, DF. Anais... Brasília, DF: Embrapa Agroenergia, 2009. 1 CD-ROM.

SANTOS, A. A. et al. Avaliação da qualidade sanitária das sementes de pinhão-manso oriundas do norte de Minas Gerais. Tropical Plant Patology, Brasília, DF, v. 34, supl., p. S230, 2009.

SHARMA, S.; KAUSHIK, J. C.; KAUSHIK, N. Fusarium moniliforme causing root rot of Jatropha. Indian Phytopathology, New Delhi, v. 54, n. 2, p. 275, 2001.

SHEPETINA, F. A.; SEVAST'YANOVA, L. B. Seed technology. In: MOSCKIM, V. A. Castor. New Delhi: Amerind, 1986. p. 175-178.

SILVA, O. F.; SOARES, A. G. Recomendações para prevenção de perdas pós-colheita do mamão. Rio de Janeiro: Embrapa Agroindústria de Alimentos, 2001.

TEIXEIRA, L. C. Potencialidades de oleaginosas para produção de biodiesel. Informe Agropecuário, Belo Horizonte, v. 26, n. 1, p. 18-27, 2005.

VANZOLINI, S. et al. Qualidade sanitária e germinação de sementes de pinhão-manso. Revista Brasileira de Sementes, Londrina, v. 32, n. 4, p. 9-14, 2010. 